\title{
Ginseng nanoparticles: a budding tool for cancer treatment
}

\author{
"Nanoparticle-mediated delivery of ginsenoside and \\ ginseng extracts could be a promising way to enhance \\ the efficacy of economically important medicinal herb \\ ginseng towards cancer treatment."
}

First draft submitted: 28 February 2017; Accepted for publication: 14 March 2017; Published online: 27 April 2017

Keywords: anticancer $\bullet$ ginsenoside $\bullet$ ginsenoside nanoconjugates $\bullet$ medicinal and functional food $\bullet$ metal nanoparticles $\bullet$ Panax ginseng $\bullet$ phytochemicals

\section{Cancer}

Cancer is one of the most incurable diseases and is a major cause of death worldwide. It is estimated that the annual number of deaths due to cancer will be around 13.2 million by 2030 in all over the world [1]. Although chemotherapy is one of the mainstays for its treatment, the side effects as well as disadvantages, such as, normal cell cytotoxicity, chemoresistance and the ability to prevent the occurrence of cancers have been considered as important hitches. Thus, the complementary and alternative medicines with the utilization of the medicinal and functional food herbs could be an alternative method to combat the progression of cancers. In this juncture, oriental adaptogenic herbs (i.e., ginseng) could be promising candidates for the treatment of cancer as safe, complementary and alternative medicines as well as medicinal and functional food $[1,2]$.

\section{Ginseng \& its relations to cancer}

Ginseng or scientifically listed in the Panax species as Panax ginseng (Korean ginseng), $P$. quinquefolius (American ginseng) and $P$. notoginseng (Sanchi ginseng) has been considered one of the highly valued medicinal plants in traditional Chinese medicine for more than thousands of years. Commonly, ginseng is also known as 'king of all herbs'. Derived from Latin, 'Panax' means 'all-healing' and 'ginseng' is literally defined as 'essence of men' with its roots resembling human-like shape [1,2]. Ginseng phytochemicals, such as, ginsenoside (unique triterpenoid saponins), phenols and acidic polysaccharides have been known to exhibit numerous pharmacological efficacies including anticancer, anti-inflammatory, antidiabetic, antiaging, enhanced immunization and liver functions and protective effects against Alzheimer's disease [3]. Their administration often results in adaptogenic effects. Regular intake of ginseng products has been demonstrated to prevent the occurrence of various cancers, ameliorate cancer-related fatigue and enhance life span [1]. Among ginseng phytochemicals, ginsenosides have been thoroughly researched and scrutinized over the years to flaunt various pharmacological activities.

\section{Limitations}

However, after oral administration, crude and major ginsenosides are mainly converted into minor ginsenosides due to hydrolysis of glucose molecules by intestinal microbiota [4]. Among minor ginsenosides, Rg3, Rh2, CK and aglycone protopanaxadiol (PPD) were already initiated in various clinical trials. Remarkably, primary metabolites reaching systemic circulations and higher uptake ratio than other major ginsenosides were reported [4]. Moreover, these compounds have been stated to possess various pharmacological and potent anticancer effects [1]. However, the clinical application

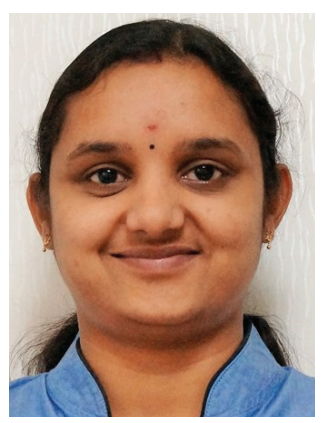

Ramya Mathiyalagan Graduate School of Biotechnology \& Ginseng Bank, College of Life Sciences, Kyung Hee University, Yongin 17104, Republic of Korea

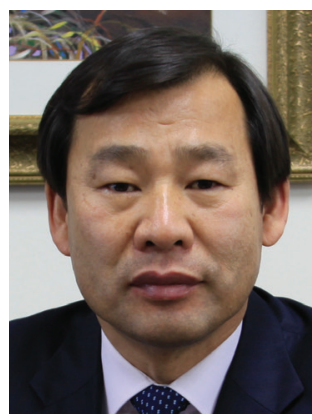

Deok Chun Yang Author for correspondence: Graduate School of Biotechnology \& Ginseng Bank, College of Life Sciences, Kyung Hee University, Yongin 17104, Republic of Korea dcyang@khu.ac.kr 
of ginsenoside is significantly hampered due to its limited solubility, low oral bioavailability and nontargeted cytotoxicity to normal cells [4-6]. As a result, biomolecular conjugations of ginsenosides and drug delivery techniques play significant roles to solve these problematic issues. Most reported nanodrug delivery carriers, such as, polymer-drug conjugates, nanoparticles, liposomes and metal nanoparticles are designed to increase solubility, improve lipid membrane penetration, enhance anticancer efficacy, ameliorate sustainability in gastrointestinal environment and reduce or eliminate loss during oral administration [6].

"Oriental adaptogenic herbs (i.e., ginseng) could
be promising candidates for the treatment of
cancer as safe, complementary and alternative
medicines as well as medicinal and functional
food."

\section{The role of ginsenoside nanoconjugates in treating cancers}

Polymer-ginsenoside nanoconjugates have been recently studied as a potential drug carrier to tumor sites owing to the improved solubility and efficient drug-release mechanisms. The enhanced oral bioavailability, oncogene $M D M 2$ targeting and anticancer activities were reported in both in vitro and in vivo of PEG-PLGA loaded 25- $\mathrm{OCH}_{3}-\mathrm{PPD}$ nanoparticles than nonloaded drug [7]. Mathiyalagan et al. have previously developed several hydrophilic polymer-ginsenoside conjugates to improve the delivery of poor water soluble ginsenosides, namely CK $[8,9]$ and PPD [5]. In that, the C-3 position of the hydroxyl group of ginsenoside was covalently conjugated to the amine group of hydrophilic glycol chitosan or carboxylic groups of carboxylated PEG through $\mathrm{pH}$-responsive linkages by carbodiimide coupling reactions. The advantage of these polymeric nanoparticles is that ginsenosides were released rapidly when conjugates were exposed in acidic condition ( $\mathrm{pH}$ 5.0), mimicking the tumor environment, inflamed tissues and cellular compartments than at physiological $\mathrm{pH}$ ( $\mathrm{pH} 7.4)$ in in vitro studies. As the prime importance, the polymer ginsenoside conjugates enhanced cell cytotoxicity in HT29 cancer cells maintaining good cell viability in murine macrophage (RAW264.7) cells than CK alone. Moreover, it inhibited lipopolysaccharide (LPS)-induced nitric oxide production [8]. Furthermore, Rg3 and PEG-PLGA-Rg3 treated Lewis lung cancer mice showed the reduced expression of cancer-related proteins. However, the significant contribution between free drugs and loaded drugs was not observed [10]. In contrast, another study of PEG-PLGA-Rg3 nanoparticles exhibited significant cytotoxicity and apoptosis [11]. Besides, the higher serum stability, better uptake, narrow-sized hybrid nanocomplex nanoparticles resulted in longer blood circulation time of $(S)-\operatorname{Rg} 3$ in in vivo pharmacokinetic study [12].

Albumin-based nanocarrier is another attractive venue for ginsenoside delivery. Yang et al. proposed a nanocapsule which is composed of human serum albumin and loaded with ginsenoside $\operatorname{Rg} 3$ with $70 \%$ efficiency. The novelty of this nanoencapsulation was the incorporation of $\mathrm{Fe}_{3} \mathrm{O}_{4}$, which magnetized the nanospheres to induce magnetic fluid hypothermia toward cervical cancer cells in vitro. The synergistic effect of chemotherapy by $\operatorname{Rg} 3$ and hyperthermia proved to be most effective at in vitro apoptosis of HeLa cervical cancer cells [13].

To improve aqueous solubility and liposolubility, $\mathrm{Yu}$ et al. attempted to prepare ginsenoside $\mathrm{Rg} 3$ and nanomicelle formulation aided by bile salt phosphatidylcholine-based system. The resulting nanomicelle formulation was optimized with high encapsulation efficiency and demonstrated in vitro inhibition of human malignant melanoma (A375). The Rg3micelle showed dose-dependent tumor inhibition with the gradual increase in drug concentration and significantly inhibited angiogenesis in the chick chorionic epithelium [14].

Finally, liposomal $\operatorname{Rg} 3$ formulation has been developed by Yu et al. to examine the activity of ginsenoside $\mathrm{Rg} 3$ in vitro against human liver cancer (HepG2) and lung cancer (A549) cell lines and in vivo pharmacokinetic behavior in Wistar rats by intravenous administration of tumor cells. As a result, the cytotoxicity and ratio of tumor inhibition of liposomal Rg3 group were significantly higher than the free Rg3 group. This liposomal formulation could potentially be utilized as a viable clinical agent to improve the anticancer activity of $\operatorname{Rg} 3$ [15].

\section{The role of metal nanoparticles mediated by $P$. ginseng phytochemicals in cancer treatment}

The phytochemicals in plant extracts have a direct relationship in the efficacy of tailor-made nanoparticles used as drug delivery and as therapeutic agents. The phytochemicals in ginseng provide binary functions in the nanoparticle synthesis as competent reducing agents to convert macrosized salts into nanosized metal nanoparticles as well as stabilizers to cater a potent coating on the metal nanoparticles [16].

Singh et al. listed the pharmacological importance of gold and silver nanoparticles mediated by $P$. ginseng fresh leaves, such as, noncytotoxicity in human keratinocytes (HaCat) and preadipocytes cells (3T3-L1), anti-inflammatory effect in LPS-induced RAW264.7 and anticancer activity in A549 [17]. Furthermore, 
ginseng leaves mediated silver nanoparticles have shown significant activity towards A549, breast cancer (MCF7) and HepG2 cells resulting in the inhibition in cell viability and oxidative stress in cancer cells without cytotoxicity in normal cells. The regulation of EGFR/p38 MAPK/p53 pathway might be the possible mechanism of its anticancer activity in A549 cells [18].

\section{Conclusion}

In short, nanoparticles of ginsenoside by various nanocarriers, such as, polymers, proteins, micelles and liposomes result in an increased water solubility and anticancer activity. In addition, the cytotoxicity of the conjugates is often similar or superior compared with bare ginsenosides in cancer cells with relatively low cytotoxicity in normal cells. The use of ginsenoside nanoconjugates could be a promising candidate against cancer and various other diseases, such as inflammation, osteoporosis and obesity in the future.

\section{Future perspective}

Nanoparticles can be efficient in cancer therapies, such as passive and active tumor targeting, gene silencing, hyperthermia, drug delivery and radiotherapy. However, there are concerns, such as their toxicity toward normal cells, degradation after delivery in vivo, their propensity to accumulate in cancer cells and half-life due to opsonization by histiocytes, blood phagocytic and reticuloendothelial cells. Nanoparticles may also accumulate at different rates in tissues and organs, such as lymph nodes, bone marrow, spleen, adrenals, liver and kidneys [19]. To overcome these disadvantages, specifically made nanoparticles for renal clearance and tumor targeting need to be developed by various parameters such as utilizing a class of ultrasmall nanoparticles and neutral/positively charged surface chemistry with differing densities. The density-dependent in vivo behavior of nanoparticles likely results from their distinct margination in laminar blood flow, which opens up a new path for precise control of nanomedicines in vivo [20].

\section{Financial \& competing interests disclosure}

This research was supported by a grant from Korean National Research Resource Center (NRF-2012M3A9B8021791), Republic of Korea. The authors have no other relevant affiliations or financial involvement with any organization or entity with a financial interest in or financial conflict with the subject matter or materials discussed in the manuscript apart from those disclosed.

No writing assistance was utilized in the production of this manuscript.

\section{References}

1 Wong AS, Che CM, Leung KW. Recent advances in ginseng as cancer therapeutics: a functional and mechanistic overview. Nat. Prod. Rep. 32(2), 256-272 (2015).

2 Kim YJ, Zhang D, Yang DC. Biosynthesis and biotechnological production of ginsenosides. Biotechnol. Adv. 33(6 Pt 1), 717-735 (2015).

3 Mohanan P, Subramaniyam S, Mathiyalagan R, Yang DC. The molecular signaling of ginsenosides Rb1, Rg1, Rg3 and their mode of actions. J. Ginseng Res. doi:http://dx.doi. org/10.1016/j.jgr.2017.01.008 (2017) (Epub ahead of print).

4 Tawab MA, Bahr U, Karas M, Wurglics M, SchubertZsilavecz M. Degradation of ginsenosides in humans after oral administration. Drug Metab. Dispos. 31(8), 1065-1071 (2003).

5 Mathiyalagan R, Kim Y-J, Wang C et al. Protopanaxadiol aglycone ginsenoside-polyethylene glycol conjugates: synthesis, physicochemical characterizations, and in vitro studies. Artif. Cells Nanomed. Biotechnol. 44(8), 1803-1809 (2016).

6 Ganesan P, Ko H-M, Kim I-S, Choi D-K. Recent trends of nano bioactive compounds from ginseng for its possible preventive role in chronic disease models. RSC Adv. 5(119), 98634-98642 (2015)

7 Voruganti S, Qin JJ, Sarkar S et al. Oral nano-delivery of anticancer ginsenoside 25-OCH3-PPD, a natural inhibitor of the $M D M 2$ oncogene: Nanoparticle preparation, characterization, in vitro and in vivo anti-prostate cancer activity, and mechanisms of action. Oncotarget 6(25), 21379-21394 (2015).

8 Mathiyalagan R, Subramaniyam S, Kim YJ, Kim YC, Yang DC. Ginsenoside compound K-bearing glycol chitosan conjugates: synthesis, physicochemical characterization, and in vitro biological studies. Carbohydr. Polym. 112, 359-366 (2014).

9 Mathiyalagan R, Subramaniyam S, Kim Y-J et al. Synthesis and pharmacokinetic characterization of a $\mathrm{pH}$-sensitive polyethylene glycol ginsenoside CK (PEG-CK) conjugate. Biosci. Biotechnol. Biochem. 78(3), 466-468 (2014).

10 Geng L, Fan J, Gao QL, Yu J, Hua BJ. Preliminary study for the roles and mechanisms of 20(R)-ginsenoside Rg3 and PEG-PLGA-Rg3 nanoparticles in the Lewis lung cancer mice. Beijing Da Xue Xue Bao 48(3), 496-501 (2016).

11 Li B, Yuan HY, Wang X et al. Preparation, release-control and cell apoptosis of $\mathrm{C}_{6}$ glioma cells in PEG-PLGA-Rg3 nanoparticles. Chem. Res. Chin. Univ. 26(5), 780-784 (2010).

12 Lee JY, Yang H, Yoon IS et al. Nanocomplexes based on amphiphilic hyaluronic acid derivative and polyethylene glycol-lipid for ginsenoside rg3 delivery. J. Pharm. Sci. 103(10), 3254-3262 (2014).

13 Yang R, Chen D, Li M, Miao F, Liu P, Tang Q. 20(s)ginsenoside Rg3-loaded magnetic human serum albumin nanospheres applied to HeLa cervical cancer cells in vitro. Biomed. Mater. Eng. 24(6), 1991-1998 (2014). 
14 Yu X, Xu H, Hu M et al. Ginsenoside Rg3 bile saltphosphatidylcholine-based mixed micelles: design, characterization, and evaluation. Chem. Pharm. Bull. 63(5), 361-368 (2015).

15 Yu H, Teng L, Meng Q et al. Development of liposomal ginsenoside Rg3: formulation optimization and evaluation of its anticancer effects. Int. J. Pharm. 450(1-2), 250-258 (2013).

16 Pérez ZEJ, Mathiyalagan R, Markus J et al. Ginseng-berrymediated gold and silver nanoparticle synthesis and evaluation of their in vitro antioxidant, antimicrobial, and cytotoxicity effects on human dermal fibroblast and murine melanoma skin cell lines. Int. J. Nanomedicine 12, 709-723 (2017).

17 Singh P, Singh H, Ahn S et al. Pharmacological importance, characterization and applications of gold and silver nanoparticles synthesized by Panax ginseng fresh leaves. Artif. Cells Nanomed. Biotechnol. doi:10.1080/21691401.2016.1243 547 (2016) (Epub ahead of print).

18 Castro-Aceituno V, Ahn S, Simu SY et al. Anticancer activity of silver nanoparticles from Panax ginseng fresh leaves in human cancer cells. Biomed. Pharmacother. 84, 158-165 (2016).

19 Conde J, Doria G, Baptista P. Noble metal nanoparticles applications in cancer. J. Drug Deliv. 2012, 12 (2012).

20 Xu J, Peng C, Yu M, Zheng J. Renal clearable noble metal nanoparticles: photoluminescence, elimination, and biomedical applications. Wiley Interdiscip. Rev. Nanomed. Nanobiotechnol doi:10.1002/wnan.1453 (2017) (Epub ahead of print). 